(1)

George Fox

UNIVERSITY
Digital Commons @ George Fox University

Faculty Publications - School of Physical

Therapy

School of Physical Therapy

2000

The Effects of the Lower Extremity Joint Motions on the Total

Body Motion in Sit-to-Stand Movement

Bing Yu

Nicole Holly-Crichlow

Paul Brichta

Gordon R. Reeves

Cynthia M. Zablotny

See next page for additional authors

Follow this and additional works at: https://digitalcommons.georgefox.edu/pt_fac

Part of the Physical Therapy Commons 
Authors

Bing Yu, Nicole Holly-Crichlow, Paul Brichta, Gordon R. Reeves, Cynthia M. Zablotny, and Deborah A. Nawoczenski 


\title{
The effects of the lower extremity joint motions on the total body motion in sit-to-stand movement
}

\author{
Bing Yu ${ }^{a, *}$, Nicole Holly-Crichlow ${ }^{\mathrm{a}}$, Paul Brichta ${ }^{\mathrm{a}}$, Gordon R. Reeves ${ }^{\mathrm{a}}$, \\ Cynthia M. Zablotny ${ }^{\mathrm{b}}$, Deborah A. Nawoczenski ${ }^{\mathrm{b}}$ \\ ${ }^{a}$ Division of Physical Therapy, The University of North Carolina at Chapel Hill, CB\#7135 Medical School Wing E, Chapel Hill, NC 27599-7135, USA \\ ${ }^{\mathrm{b}}$ Department of Physical Therapy, Ithaca College, University of Rochester Campus, Rochester, NY, USA
}

\begin{abstract}
Objective. The purpose of this study is to investigate the effects of lower extremity joint angular motions on the whole body linear motions in a sit-to-stand movement using a biomechanical model that describes the whole body linear velocity vector as functions of lower extremity joint angular velocities.

Design. Two-dimensional video analysis of whole body and joint kinematics.

Background. A biomechanical model that describes the whole body linear motions as functions of lower extremity joint angular motions is needed to provide clinically relevant information in clinical services and scientific research.

Methods. The linear velocity vector of the whole body motion during the sit-to-stand movement was partitioned into horizontal and vertical components and expressed as functions of lower extremity joint angular velocities for 10 healthy subjects. The coefficient of joint contribution to the whole body linear velocity vector was determined for each joint in each direction.

Results. The ankle and hip angular motions are critical to the development of the forward horizontal velocity of the whole body during the sit-to-stand movement. The knee and hip angular motions are critical to the development of the upward vertical velocity of the whole body during the sit-to-stand movement.

Conclusions. The hip, knee, and ankle joint angular motions have various roles in whole body motions in different directions of the sit-to-stand movement.

\section{Relevance}

The model and the results of this study can be applied to study the control strategies, falls, and assessments of functional impairments in the sit-to-stand movement. (C) 2000 Elsevier Science Ltd. All rights reserved.
\end{abstract}

Keywords: Sit-to-stand; Lower extremity; Joint angular motion; Whole body linear motion; Control strategies; Fall; Functional impairment

\section{Introduction}

Standing up from a seated position is an important functional task in daily living. It is an essential prerequisite to movement in an upright posture. Completing this seemingly simple task requires a complex coordination of the central nervous system and neuromuscular system [1]. Quantification of the biomechanical factors that underlie the ability to rise from a chair is important for addressing the control strategies

\footnotetext{
"Corresponding author.

E-mail address: bing.yu@css.unc.edu (B. Yu).
}

that may impact the successful completion of the sit-tostand (STS) movement.

The control strategies responsible for the directionspecific differences in the maximum momentum of the whole body during the STS movement have been assessed using different biomechanical models. The outcomes of these models characterized the effects of segment angular motions on the linear motions of the whole body during the STS movement. These effects were referred to as segmental contributions to the linear motions of the whole body. Using a four-segment model based on a mathematical model proposed by Ae et al. [2], Pai and Rodgers [1] found that the head-arm-trunk segment was the major contributor to the maximum horizontal momentum of the whole body during the 
STS movement. They also found that the thigh segment was the major contributor to the maximum vertical momentum of the whole body and responsible for the change in the magnitude of the maximum vertical momentum of the whole body corresponding to the change in the speed of the STS movement [3]. After analyzing the absolute momentum of different body segments in STS movement, Riley et al. [4] found that the upper body was the major contributor to the maximum momentum of the whole body in both the horizontal and vertical direction.

Although the mathematical model proposed by Ae et al. [2] has been frequently used to describe the effects of segment angular motions on the linear motions $[1,5,6]$, it does not provide information about the effects of joint angular motion on direction-specific differences in whole body linear motion. A description of segment movement provides information about the movement of a segment or number of segments relative to a defined external or laboratory reference frame. A description of joint angular motion provides information on how two articulated segments move relative to each other. Joint angular motion is the basic form of human body motions from which segment and total body movements are derived [7]. When assessing impairments that may lead to movement dysfunction, clinicians routinely focus on joint angular motion. A model that describes whole body linear motion in terms of joint angular motions provides clinically relevant information for analysis of whole body movement and additional insight into the development of joint-specific treatment strategies.

The purpose of this study is to investigate the effects of lower extremity joint angular motions on the whole body linear motions of normal young healthy adults in an STS movement using a new model that expresses whole body linear velocity vector as a function of joint angular velocities. It is hypothesized: (a) that the effects of different lower extremity joint angular motions on the whole body linear motion in a given direction are different, and (b) that the effects of a given lower extremity joint angular motion on the whole body linear motions in different directions are different.

\section{Methods}

Ten healthy young adults (five males and five females) without any history of lower extremity disorders were recruited as the subjects. The mean age, height, and body mass of these subjects were 31.1 (S.D. 7.4) yr, 1.72 (S.D. 0.11 ) $\mathrm{m}$, and 73.3 (S.D. 16.3) kg, respectively. The use of human subjects in this study was approved by the University of North Carolina at Chapel Hill school of Medicine Committee on the Protection of the Rights of Human Subjects. Written consent was obtained from each subject.
An S-VHS video camcorder was used to record the right side-view of each subject's movement at a frame rate of $60 \mathrm{~Hz}$. It was assumed in this study that the STS movement is primarily two-dimensional and occurs in a vertical plane perpendicular to the optical axis of the camcorder and through the midpoint of the line connecting subject's toes. Two reflective markers were placed $1.7 \mathrm{~m}$ apart along a horizontal line on the floor in the assumed motion plane to assist in converting the two-dimensional video coordinates to two-dimensional real-life coordinates.

Each subject was tested barefoot. Seven reflective markers were placed at the right acromion process, right olecranon process, right greater trochanter, right lateral tibial condyle, right lateral malleolus, the posterior calcaneum, and the dorsum of the right great toe (Fig. 1). A flat wooden bench $458 \mathrm{~mm}$ high was used as the seat, placed on the horizontal reference scale with one surface parallel to the assumed motion plane. The height of the floor was adjusted so that the knee was at $90^{\circ}$ flexion and the shank was perpendicular to the floor when the subject was seated (Fig. 1). The subject performed the STS movement at a self-selected comfortable speed with the arms folded in front of the body. Ten successful trials were recorded for each subject.

The videotape record of the subjects' performances was digitized at a sampling rate of $60 \mathrm{~Hz}$ using a PEAK

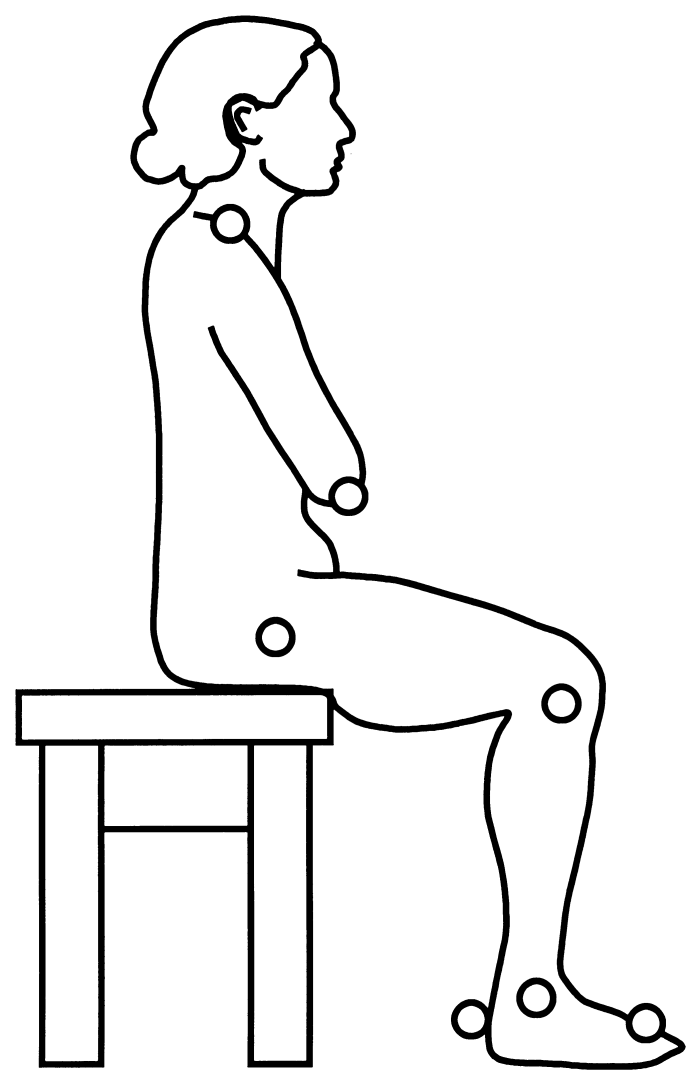

Fig. 1. The starting position of the STS movement tested in this study. 
Performance video analysis system. The two-dimensional digitized video coordinates of the reflective markers were converted to real-life two-dimensional coordinates. The real-life coordinates were filtered through a fourth-order Butterworth digital filter [8] at a cutoff frequency of $3 \mathrm{~Hz}$ [1].

A 14-segment model was used to represent each subject's body. The 14 body segments included: head, trunk, left and right upper arms, left and right forearms, left and right hands, left and right thighs, left and right shanks, and left and right feet. The position and orientation of the head relative to the trunk was estimated from the subject's standing height, the vertical coordinate of the reflective mark on the acromion process, and the position and orientation of the trunk. The segmentation method [9] was used to determine the locations of the center of mass (CoM) of each segment and the whole body. The forearms and hands were treated as mass particles at the center of the elbow joint because the forearms were folded in front of the trunk and approximately perpendicular to the image plane of the camcorder. Segment inertia data of Hinrichs [10] were used to determine the relative mass and location of the CoM for each segment. The CoM of the head-armtrunk (HAT) system was also determined. The HAT was considered as one segment when determining effects of joint angular motions on the whole body linear motions in the STS movement [1].

The linear velocity vectors of the CoM of the whole body due to the angular motions of the ankle, knee, and hip joints during the STS movement were determined using

$$
\begin{aligned}
\mathbf{v}_{\mathrm{B}, \mathrm{a}}= & m_{\mathrm{S}} \omega_{\mathrm{a}} \times \mathbf{r}_{\mathrm{s} / \mathrm{a}}+m_{\mathrm{T}} \omega_{\mathrm{a}} \times\left(\mathbf{l}_{\mathrm{k} / \mathrm{a}}+\mathbf{r}_{\mathrm{t} / \mathrm{k}}\right) \\
& +m_{\mathrm{HAT}} \omega_{\mathrm{a}} \times\left(\mathbf{l}_{\mathrm{k} / \mathrm{a}}+\mathbf{l}_{\mathrm{h} / \mathrm{k}}+\mathbf{r}_{\mathrm{HAT} / \mathrm{h}}\right), \\
\mathbf{v}_{\mathrm{B}, \mathrm{k}}= & m_{\mathrm{T}} \omega_{\mathrm{k}} \times \mathbf{r}_{\mathrm{t} / \mathrm{k}}+m_{\mathrm{HAT}} \omega_{\mathrm{k}} \times\left(\mathbf{l}_{\mathrm{h} / \mathrm{k}}+\mathbf{r}_{\mathrm{HAT} / \mathrm{h}}\right), \\
\mathbf{v}_{\mathrm{B}, \mathrm{h}}= & m_{\mathrm{HAT}} \omega_{\mathrm{h}} \times \mathbf{r}_{\mathrm{HAT} / \mathrm{h}},
\end{aligned}
$$

where $\mathbf{v}_{\mathrm{B}, \mathrm{a}}, \mathbf{v}_{\mathrm{B}, \mathrm{k}}$, and $\mathbf{v}_{\mathrm{B}, \mathrm{h}}$ are instantaneous linear velocity vectors of the CoM of the whole body due to the angular motions of the ankle, knee, and hip joints, respectively; $m_{\mathrm{S}}, m_{\mathrm{T}}$, and $m_{\mathrm{HAT}}$ are relative masses of the shank, thigh, and HAT; $\omega_{\mathrm{a}}, \omega_{\mathrm{k}}$, and $\omega_{\mathrm{h}}$ are angular velocity vectors of the ankle, knee, and hip joints, respectively, that were defined as the angular velocities of the shank relative to the foot, of the thigh relative to the shank, and of the trunk relative to the thigh, respectively; $\mathbf{l}$ is the location vector of the proximal joint of a given segment relative to the distal joint of the same segment, and $\mathbf{r}$ is the location vector of the CoM of a given segment relative to the distal joint of the same segment. The assumptions in the above described calculations are: (a) the STS movement occurs in a twodimensional plane and is bilaterally symmetric [11], (b) the ankle, knee, and hip joints are hinge joints, and (c) the head, arms, and trunk do not move relative to each other during the STS movement defined in this study.

The velocity vectors of the CoM due to joint angular motions were decomposed to horizontal and vertical components. The last frame in which the horizontal velocity of the CoM was zero before it reached its peak value was considered as the beginning of the STS movement. The first frame in which the vertical velocity of the CoM was zero after it reached its peak value was considered as the end of the STS movement. A trial was considered successful if the CoM of the whole body moved continuously throughout the STS movement cycle and had a zero velocity vector before the beginning of the movement and after the end of the movement. The time period between the beginning and end of the STS movement is defined as a full STS movement cycle. The STS movement cycle in each trial was divided into 100 intervals that were normalized to the time of the full STS movement cycle. The first five successful trials were normalized and averaged for each subject.

The signs of horizontal and vertical velocity components indicate the direction of the movements in the horizontal and vertical directions. In this study, a positive horizontal velocity indicates a forward movement while a negative horizontal velocity indicates a backward movement. A positive vertical velocity indicates an upward movement while a negative vertical velocity indicates a downward movement.

To examine the effects of a given joint angular motion on the whole body linear motions, linear velocities of the $\mathrm{CoM}$ without the given joint angular motion were determined as

$$
\begin{aligned}
& \mathbf{v}_{\mathrm{B} / \mathrm{a}}=\mathbf{v}_{\mathrm{B}}-\mathbf{v}_{\mathrm{B}, \mathrm{a}}, \\
& \mathbf{v}_{\mathrm{B} / \mathrm{k}}=\mathbf{v}_{\mathrm{B}}-\mathbf{v}_{\mathrm{B}, \mathrm{k}}, \\
& \mathbf{v}_{\mathrm{B} / \mathrm{h}}=\mathbf{v}_{\mathrm{B}}-\mathbf{v}_{\mathrm{B}, \mathrm{h}},
\end{aligned}
$$

where $\mathbf{v}_{\mathrm{B}}$ is the velocity vector of the whole body CoM that is estimated from changes in locations of the whole body CoM and sample rate using central finite difference method, and $\mathbf{v}_{\mathrm{B} / \mathrm{a}}, \mathbf{v}_{\mathrm{B} / \mathrm{k}}$, and $\mathbf{v}_{\mathrm{B} / \mathrm{h}}$ are velocity vectors of the CoM in STS movement without ankle, knee, and hip joint angular motions, respectively.

A parameter named as the coefficient of joint contribution was used as a measure of the effect of a given joint angular motion on the whole body linear motion in a given direction during the entire STS movement cycle. The coefficient of joint contribution for a given joint $j$ can be calculated using

$$
\mathrm{CJC}_{j}=\frac{\sum_{t=0}^{T} v_{\mathrm{B}, j, t}^{2}}{\sum_{t=0}^{T}\left(v_{\mathrm{B}, \mathrm{a}, t}^{2}+v_{\mathrm{B}, \mathrm{k}, t}^{2}+v_{\mathrm{B}, \mathrm{h}, t}^{2}\right)},
$$

where $v_{\mathrm{B}, j, t}$ is the velocity of the CoM in a given direction due to the angular motion of joint $j$ at time $t$, and 
$T$ is the total number of intervals in a normalized STS movement cycle, which was 100 in this study.

The coefficient of joint contribution is a parameter that describes the similarity of the velocity-time curve of the whole body $\mathrm{CoM}\left(\mathbf{v}_{\mathrm{B}}\right)$ to the velocity-time curve of the whole body CoM due to the angular motion of joint $j\left(\mathbf{v}_{\mathrm{B}, j}\right)$. It represents the percentage of the total variation of the velocity of the whole body CoM that is due to a given joint angular motion. The numerator of the ratio on the right-hand side of Eq. (3) is the variation of the velocity generated by the angular motion of joint $j$. The denominator is the total variation of the velocities generated by the angular motions of all joints considered in this study. The greater the magnitude of the coefficient of joint contribution for a given joint, the greater the effect of the given joint angular motion on the linear motion of the whole body CoM. The coefficient of joint contribution for the given joint is zero if the velocity of the $\mathrm{CoM}$ in a given direction due to a given joint angular motion is zero throughout the entire movement cycle. This means that the given joint angular motion has no effect on the linear motion of the CoM in the given direction.

A $3 \times 2$ two-way ANOva with repeated measures was conducted with the coefficient of joint contribution as the dependent variable and joint and direction as independent variables. In case of significant differences, paired $t$-tests with Bonferroni procedure were conducted to locate the differences. The $5 \%$ level of confidence was chosen to indicate overall statistical significance. A maximum of three paired $t$-tests might need to be conducted for each direction. The adjusted level of confidence for paired $t$-tests using Bonferroni procedure was $1 \%$ for a possible maximum of six paired $t$-tests.

\section{Results}

A significant joint effect $(P<0.001)$ and a significant interaction effect of joint and direction of the magnitude of the coefficient of joint contribution $(P<0.001)$ were found in the ANOva. The follow-up paired $t$-tests revealed that the magnitude of the coefficient of joint contribution of the knee joint to the horizontal velocity of the CoM was significantly greater than those of the ankle and hip joints $(P<0.001)$ (Fig. 2). There was no significant difference in the magnitude of the coefficient of joint contribution to the horizontal velocity of the CoM between the ankle and hip joints. The follow-up $t$-tests also revealed that a significant increase in the coefficient of knee joint contribution to the vertical velocity of the CoM in comparison to that of the ankle joint, and a significant increase in the coefficient of hip joint contribution to the vertical velocity of the CoM in comparison to the knee joint $(P<0.001)$ (Fig. 2).

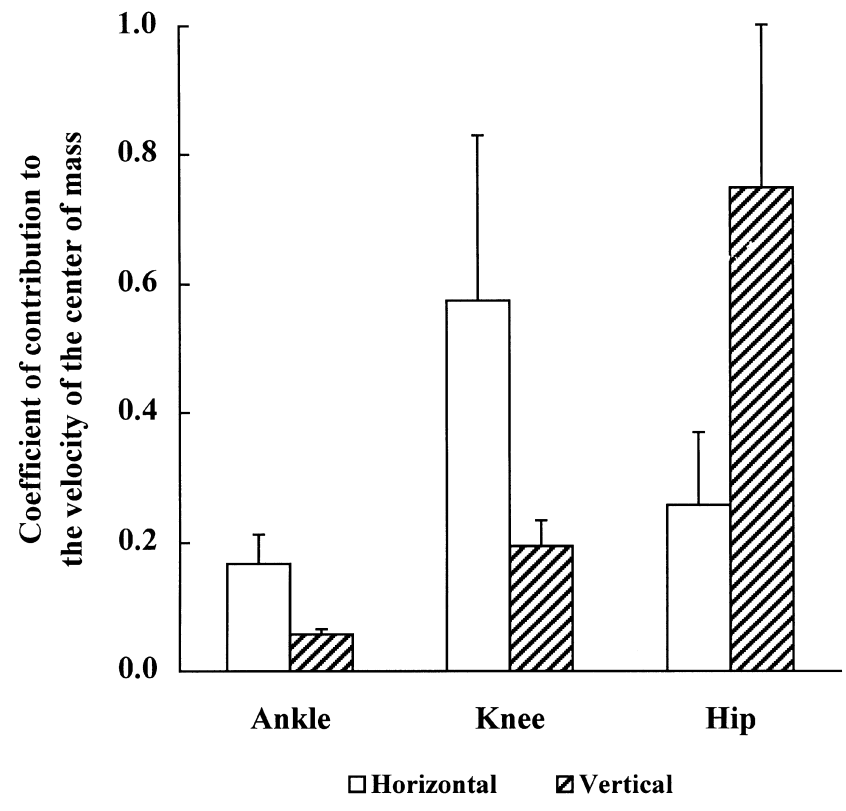

Fig. 2. The joint contribution coefficients in the STS movement.

The forward horizontal velocity of the CoM reached its maximum of 0.61 (S.D. 0.10) $\mathrm{m} / \mathrm{s}$ at $29 \%$ of the STS movement cycle (Fig. 3). After reaching its maximum, the horizontal velocity of the $\mathrm{CoM}$ decreased continuously until reaching zero at the end of the movement cycle (Fig. 3).

The forward horizontal velocity of the CoM without hip joint angular motion reached 0.3 (S.D. 0.14 ) $\mathrm{m} / \mathrm{s}$ at $29 \%$ of the STS movement cycle when the total forward horizontal velocity of the CoM reached its maximum (Fig. 3(a)). This forward horizontal velocity reached its maximum of 0.56 (S.D. 0.07) $\mathrm{m} / \mathrm{s}$ at $69 \%$ of the movement cycle and then continuously decreased to zero (Fig. 3(a)). The waveform of the horizontal velocity of the CoM without knee joint angular motion was similar to a sine wave (Fig. 3(b)). This horizontal velocity reached its forward maximum of 0.61 (S.D. 0.11 ) $\mathrm{m} / \mathrm{s}$ at $30 \%$ of the STS movement cycle, and its backward maximum of 0.84 (S.D. 0.11 ) $\mathrm{m} / \mathrm{s}$ at $78 \%$ of the movement cycle (Fig. 3(b)). The forward horizontal velocity of the CoM without the ankle joint angular motion had two peaks. This forward horizontal velocity was 0.35 (S.D. 0.05 ) $\mathrm{m} / \mathrm{s}$ at the $25 \%$ of the STS movement cycle (Fig. 3(c)). The second peak of the forward horizontal velocity of the CoM without the ankle joint angular motion was 0.39 (S.D. 0.08 ) $\mathrm{m} / \mathrm{s}$ and occurred at $80 \%$ of the STS movement cycle while the total forward horizontal velocity of the CoM was approaching to zero (Fig. 3(c)).

The vertical velocity of the CoM was in a downward direction after the STS movement was started (Fig. 4). This vertical velocity reached its downward maximum of 0.05 (S.D. 0.05 ) $\mathrm{m} / \mathrm{s}$ at $31 \%$ of the STS movement cycle. This vertical velocity then changed rapidly into upward direction and reached its upward maximum of 


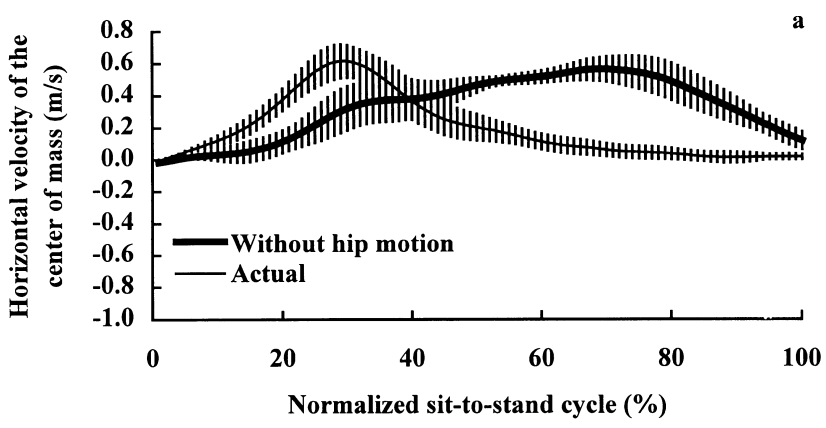

b

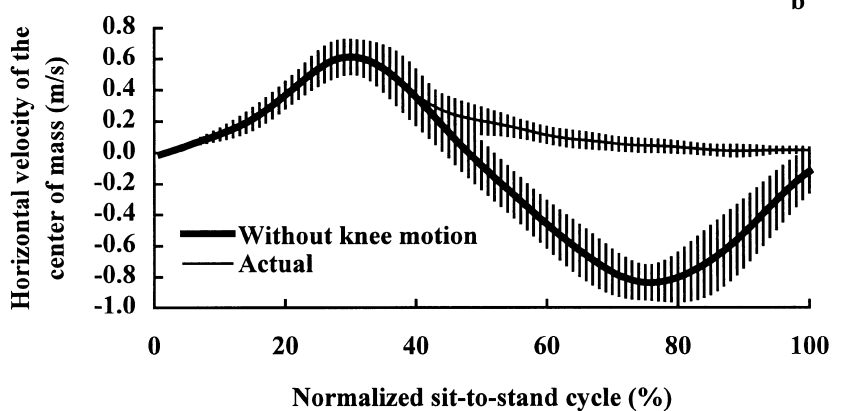

c

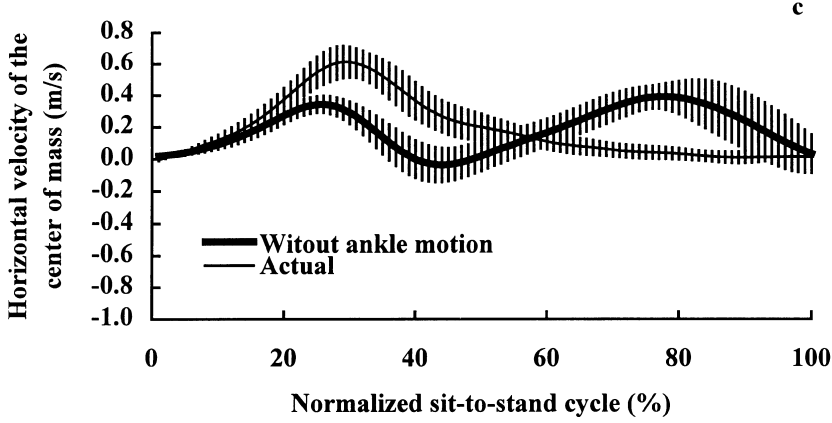

Fig. 3. The actual horizontal velocity of the CoM and the estimated horizontal velocity of the CoM without: (a) hip joint angular motion, (b) knee joint angular motion, and (c) ankle joint angular motion in the STS movement.

0.39 (S.D. 0.04 ) $\mathrm{m} / \mathrm{s}$ at $62 \%$ of the STS movement cycle (Fig. 4). After reaching the upward maximum, the vertical velocity of the CoM decreased continuously until reaching zero at the end of the movement cycle (Fig. 4).

The vertical velocity of the CoM without the hip joint angular motion was generally in upward direction. This vertical velocity had two peaks of 0.08 (S.D. 0.04 ) $\mathrm{m} / \mathrm{s}$ and 0.14 (S.D. 0.05 ) $\mathrm{m} / \mathrm{s}$ at $29 \%$ and $64 \%$ of the STS movement cycle, respectively (Fig. 4(a)). The second peak value is about $65 \%$ lower than the maximum of the total upward vertical velocity of the CoM. The general pattern of the vertical velocity of the CoM without knee joint angular motion was very similar to that of the actual vertical velocity of the CoM (Fig. 4(b)). However, the upward maximum of the vertical velocity of the CoM without the knee joint angular motion was 0.26 (S.D. 0.04 ) $\mathrm{m} / \mathrm{s}$ at $62 \%$ of the STS movement cycle, which was $35 \%$ lower than that of the total upward
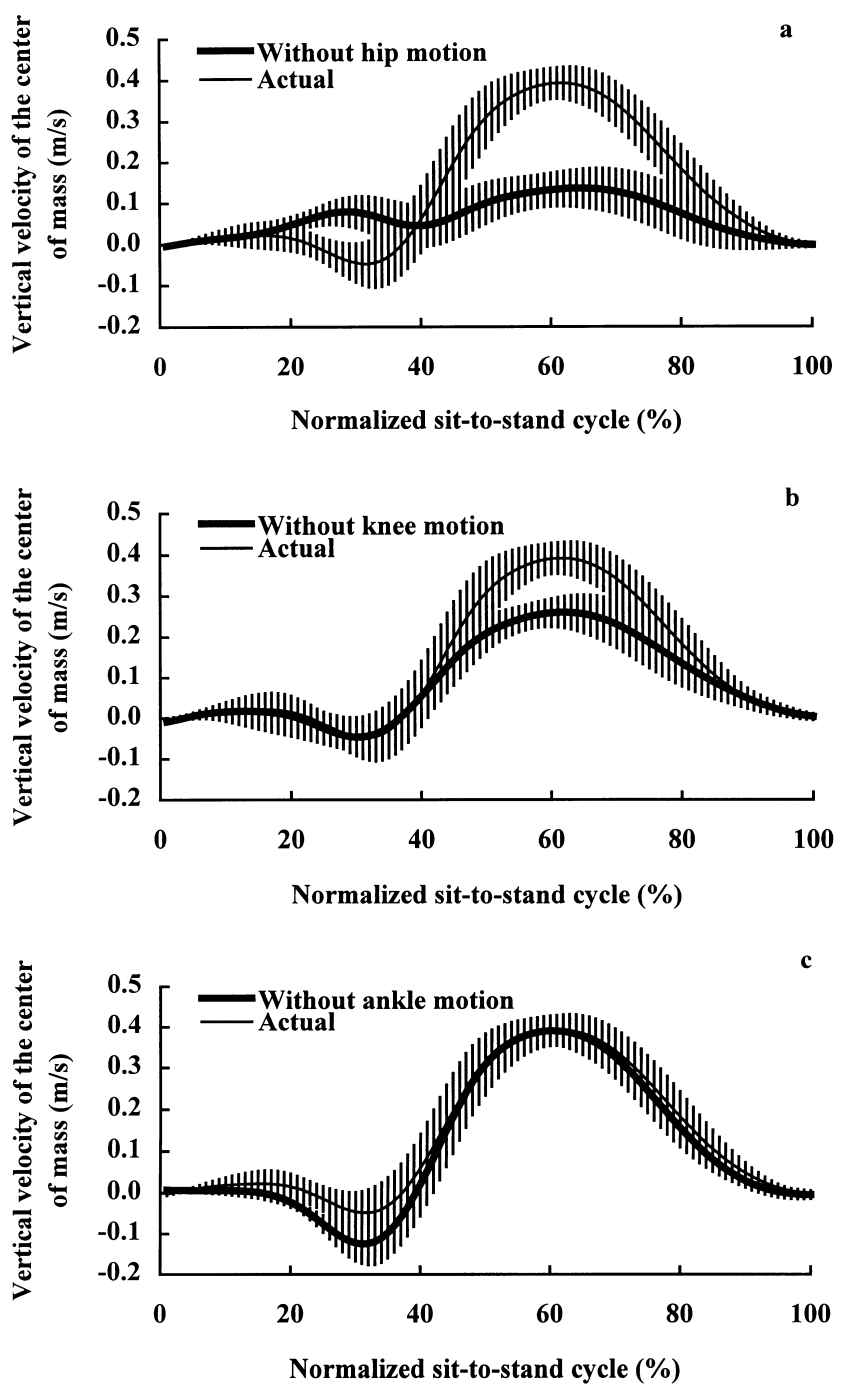

Fig. 4. The actual vertical velocity of the CoM and the estimated vertical velocity of the CoM without: (a) hip joint angular motion, (b) knee joint angular motion, and (c) ankle joint angular motion in the STS movement.

vertical velocity of the CoM. The vertical velocity of the CoM without the ankle joint angular motion was almost identical to the total velocity of the CoM (Fig. 4(c)).

\section{Discussion}

The general patterns of the total velocities of the CoM during the STS movement obtained in this study are consistent with those previously reported in the literature $[1,4,12]$. The mean forward maximum horizontal velocity of the CoM in this study is comparable to $0.59 \mathrm{~m} / \mathrm{s}$ presented by Riley et al. [4]. The mean maximum upward vertical velocity of the CoM in this study is comparable to $0.35 \mathrm{~m} / \mathrm{s}$ obtained by Roebroeck et al. [12]. The discrepancies among different studies are within the range of inter-subject variations in the STS 
movement observed in the present study. These intersubject variations can be attributed to differences in: (a) initial position of the STS movement, (b) speed of the STS movement, and (c) movement control strategies used by subjects.

The results of this study support the hypotheses of this study. The significant joint effect and significant interaction effect of joint and directions revealed by the ANOVA suggest that the effects of lower extremity joint angular motions on whole body linear motions are joint and direction specific. These results indicate that the hip, knee, and ankle joint angular motions have various roles in the control of the whole body linear motions in different directions of the STS movement.

The angular motions of all three lower extremity joints had significant effects on the linear motion of the whole body in the horizontal direction. However, as indicated by follow-up $t$-tests on the coefficient of joint contribution to the horizontal velocity of the CoM, the effect of knee joint angular motion was significantly greater than those of the ankle and hip joint angular motions (Fig. 2). The effect of knee angular motion on the horizontal velocity of the CoM was mainly in the later portion of the STS movement. During the early portion of the STS movement, the maximum horizontal velocity of the CoM without the knee angular motion (Fig. 3(c)) was essentially identical to the maximum actual horizontal velocity of the CoM (Fig. 3(a)). This indicates that the whole body linear motion would still be able to obtain enough forward horizontal velocity during the early part of the STS movement even if the knee joint angular motion were absent. However, during the later phase of the STS movement, the horizontal velocity of the CoM without the knee joint angular motion was in the backward direction (Fig. 3(c)) while the corresponding actual horizontal velocity of the CoM was close to zero and in the forward direction (Fig. 3(a)). Theoretically, this indicates that the subject could fall backward if the knee joint angular motion was absent and no compensation was made at other joints.

The maximum forward horizontal velocity of the CoM during the STS movement was primarily obtained through ankle and hip joint angular motions. The peak forward horizontal velocity of the CoM without ankle or hip joint angular motion was about $50 \%$ lower than the actual horizontal velocity of the CoM during this portion of the STS movement (Figs. 3(b) and (d)). These results indicate that the whole body would not be able to obtain sufficient forward horizontal velocity during the early portion of the STS movement if either the ankle or hip joint angular motion were absent or insufficient. Further, during the later portion of the STS movement, the forward horizontal velocity of the CoM without ankle or hip joint motion was significantly greater than the actual total forward horizontal velocity of the CoM. Theoretically, this indicates that the subject could fall forward if the ankle or hip joint motion was absent and no compensation was made at other joints.

All three lower extremity joint angular motions also have significant effects on the whole body linear motion in the vertical direction in the STS movement. The effect of the ankle joint angular motion on the whole body linear motion in the vertical direction is mainly reflected by the upward vertical velocity it provided during the early portion of the STS movement. The upward vertical velocity of the $\mathrm{CoM}$ without ankle joint angular motion was lower than the actual upward vertical velocity of the CoM during the early portion of the STS movement (Fig. 4(a)). This means that the upward vertical velocity of the CoM would be lower than what is actually needed if the ankle joint angular motion were absent or insufficient during this portion of the movement. However, the ankle angular motion had little effect on the maximum and overall upward vertical velocity of the CoM.

The knee and hip angular motions have major effects on the vertical velocity of the CoM during the STS movement. The results show that the maximum upward vertical velocity of the $\mathrm{CoM}$ without the knee angular motion was about $35 \%$ lower than the maximum actual upward vertical velocity of the CoM during the later portion of the STS movement. In addition, the maximum upward vertical velocity of the CoM without the hip joint angular motion was about $65 \%$ lower than the maximum actual upward vertical velocity of the CoM during the same time period. These results indicate that the knee and hip joint angular motions provide about $35 \%$ and $65 \%$, respectively, of the maximum total upward vertical velocity of the whole body during the later portion of the STS movement (Figs. 4(b) and (c)). This implies that the maximum upward vertical velocity of the whole body would be significantly lower than what is needed for a successful STS movement if knee or the hip joint angular motion were absent. The insufficient upward vertical velocity may force the subject to sit back in the seat and thus results in an unsuccessful STS movement.

The three assumptions in the model do not have significant effects on the results. The sum of $\mathbf{v}_{\mathrm{B}, \mathrm{a}}, \mathbf{v}_{\mathrm{B}, \mathrm{k}}$, and $\mathbf{v}_{\mathrm{B}, \mathrm{h}}$ obtained in this study was essentially equal to $\mathbf{v}_{\mathrm{B}}$ with random errors less than $0.05 \mathrm{~m} / \mathrm{s}$ in both directions throughout entire movement cycle. This means that the relative motions between the head, trunk, and the upper extremities, the asymmetric motions between the right and left lower extremities, and the translations between two articulated segments did not have significant effects on the linear velocities of the CoM in the STS movement in this study. This result supports the validity of the three assumptions in this study. In addition, the assumption that the upper extremity motions have no contribution to the total body motion is specific for this study, not a general assumption of the model. The 
effects of upper extremity motions on the total body motion can be included in the model if it is necessary.

The model developed in this study is different from those used by Pai and Rodgers [1] and Riley et al. [4]. The model used by Riley et al. [4] determines segment contributions to whole body motions based on segment absolute linear motions. The model used by Pai and Rodgers [1] determines segment contributions to whole body motions based on the segment absolute angular motions. The model developed in this study determines joint contributions to whole body motions based on joint angular motions. Segment absolute linear and angular motions are not basic forms of human body motion and can be decomposed to joint angular motions that are the basic forms of human body motion. The results of this study should not be compared to those by Pai and Rodgers [1] and Riley et al. [4] because of these fundamental biomechanical differences between different models.

The model developed in this study determined only the kinematic contribution of the angular motion of a given joint to the whole body linear motions without considering the effects of the change in the given joint motion on other joint motions. The change in the angular motion of a given joint may affect the angular motions of other joints and thus their contribution to the whole body linear motions. The model developed in this study alone does not have the ability to determine this effect. The effect of a joint angular motion on the whole body linear motions determined using this model shows only the possible results of the whole body linear motion when the joint angular motion is absent, which does not include the compensation made by the other joints. The kinematic nature of the model should be kept in mind when interpreting the results based on this model.

The model developed in this study can be applied to different phases of STS movement in this study. As a previous study [12] suggested, the entire STS movement can be divided into three phases. These three phases include: (a) the acceleration phase, that is from the beginning of the movement to instant when the horizontal velocity of the CoM reaches its maximum, (b) the transition phase, that is from the instant when the horizontal velocity of the CoM reaches its maximum to the instant when the vertical velocity reaches its maximum, and (c) the deceleration phase, that is from the instant when the vertical velocity of the CoM reaches its maximum to the end of the movement. When the model is applied to each of these three phases, the coefficients of joint contribution will provide an understanding of the detailed roles of a given joint angular motion to the whole body linear motion in different phases of the STS movement.

\section{Conclusions}

This study developed a biomechanical model for evaluating kinematic effects of joint angular motions on the whole body linear motions during the STS movement. The ankle and hip angular motions are critical to the development of the forward horizontal velocity of the whole body during the early portion of the STS movement. The knee and hip angular motions are critical to the development of the upward vertical velocity of the whole body during the STS movement. All three lower extremity joint angular motions have significant effects on the horizontal motion of the whole body during the later portion of the STS movement.

\section{References}

[1] Pai YC, Rogers MW. Segmental contributions to total body Momentum in sit-to-stand. Med Sci Sports Exerc 1991;23:225-30.

[2] Ae M, Shibukawa K. A biomechanical method for the analysis of the body segments in human movement. Japan J Phys Educ 1980;25:233-43.

[3] Pai YC, Rogers MW. Speed variation and resultant joint torques during sit-to-stand. Arch Phys Med Rehabil 1991;72:881-5.

[4] Riley PO, Mann RW, Hodge WE, Hodge WA. Mechanics of a constrained chair-rise. J Biomech 1991;24:77-85.

[5] Ae M, Shibukawa K, Yokoi T, Hahihara Y. Body segment contributions during the support phase while running at different velocities, Biomechanics IX-B. Champaign, IL: Human Kinetics Publishers, 1983.

[6] Hinrichs RN. Upper extremity function in running. Doctoral dissertation, Pennsylvania State University, 1982.

[7] Yu B. Doctoral dissertation, The University of Iowa, Iowa City, Iowa, 1993.

[8] Winter DA, Sidewall HG, Hobson DA. Measurement and reduction of noise in kinematics of locomotion. J Biomech $1974 ; 7: 157-9$

[9] Hay JG. The Biomechanics of Sports Techniques. Englewood Cliffs, NJ: Prentice-Hall, 1993.

[10] Hinrichs RN. Adjustments to the segment center of mass proportions of Clauser et al. J Biomech 1990;23:949-51.

[11] Lundin TM, Grabiner MD, Jahnigen DW. On the assumption of bilateral lower extremity joint moment symmetry during the sitto-stand task. J Biomech 1995;28:109-12.

[12] Roebroeck ME, Doorenbosch CAM, Harlaar J, Jacobs R, Lankhorst JG. Biomechanics and muscular activity during sitto-stand transfer. Clin Biomech 1994;9:235-44. 